\title{
A REMARK ON THE REEB FLOW FOR SPHERES
}

\author{
ROGER CASALS AND FRANCISCO PRESAS
}

\begin{abstract}
We prove the non-triviality of the Reeb flow for the standard contact spheres $\mathbb{S}^{2 n+1}$, $n \neq 3$, inside the fundamental group of their contactomorphism group. The argument uses the existence of homotopically non-trivial 2-spheres in the space of contact structures of a 3-Sasakian manifold.
\end{abstract}

Let $(M, \xi)$ be a closed contact manifold. Consider the space $\mathcal{C}(M, \xi)$ of contact structures isotopic to $\xi$. This space has been studied in special cases. See [El] for the 3-sphere and [Bo], [Ge] for torus bundles. In the present note we prove the non-triviality of its second homotopy group for 3-Sasakian manifolds, see [BG].

Theorem 1. Let $(M, \xi)$ be a 3-Sasakian manifold, then $\operatorname{rk}\left(\pi_{2}(\mathcal{C}(M, \xi))\right) \geq 1$.

Let $\left(\mathbb{S}^{4 n+3}, \xi_{0}=\operatorname{ker} \alpha_{0}\right)$ be the standard contact sphere with the standard contact form. The nontrivial spheres in $\mathcal{C}\left(\mathbb{S}^{4 n+3}, \xi_{0}\right)$ allow us to answer a question posed in [Gi]:

Remarque 2.10: On peut se demander s'il n'y a pas, dans $\operatorname{Cont}\left(\mathbb{S}^{2 n+1}, \xi_{0}\right)$, un lacet positif contractile plus simple que dans $\mathbb{P} U(n, 1)$ et par exemple si le lacet $\rho_{t}, t \in \mathbb{S}^{1}$, n'est pas contractile. C'est peu probable mais je n'en ai pas la preuve.

The answer we provide is the following

Corollary 2. The class in $\pi_{1}\left(\operatorname{Cont}\left(\mathbb{S}^{2 n+1}, \xi_{0}\right)\right)$ generated by the Reeb flow of $\alpha_{0}$ is a non-trivial element of infinite order for $n \neq 3$.

In Section 1 we introduce the objects of interest and necessary notation. The geometric construction underlying the results is explained in Section 2. It is a generalization to higher dimensions of ideas found in [Ge]. Theorem 1 is concluded. Section 3 contains the argument deducing Corollary 2. Section 4 extends the results to higher homotopy groups.

Acknowledgements: We are grateful to V. Ginzburg for useful discussions. The first author thanks O. Spáčil for valuable remarks.

\section{Preliminaries}

\subsection{Contact structures.}

Definition 3. Let $M^{2 n+1}$ be a smooth manifold. A codimension-1 regular distribution $\xi$ is a contact distribution if there exists a 1 -form $\alpha \in \Omega^{1}(M)$ such that $\operatorname{ker} \alpha=\xi$ and $\alpha \wedge d \alpha^{n}$ is a volume form.

The structure described above is known as a cooriented contact structure. Since the non-coorientable case is not considered in this article, we refer to a cooriented contact structure simply as a contact structure. The smooth manifold $M$ will be assumed to be oriented. The contact structures to be considered will be positively cooriented, i.e. the induced orientation coincides with that prescribed on $M$.

The definition is independent of the choice of 1 -form $\alpha^{\prime}=e^{f} \alpha$, for any $f \in C^{\infty}(M, \mathbb{R})$. Let $\operatorname{Cont}(M, \xi)=\left\{s \in \operatorname{Diff}(M): d s_{*} \xi=\xi\right\}$ be the space of diffeomorphisms that preserve the contact structure. These diffeomorphisms are called contactomorphisms. The connected component of the identity of $\operatorname{Cont}(M, \xi)$ will be denoted by $\operatorname{Cont}_{0}(M, \xi) . \mathcal{C}(M, \xi)$ will stand for the space of positive contact structures in $M$ isotopic to $\xi$. The unique vector field $R$ such that

$$
i_{R} \alpha=1, \quad i_{R} d \alpha=0,
$$

2010 Mathematics Subject Classification. Primary: 53D10.

Key words and phrases. contact structures, Reeb flow. 
is called the Reeb vector field associated to $\alpha$.

A vector field $X \in \Gamma(T M)$ preserves the contact structure if it satisfies the following pair of equations

$$
\begin{aligned}
i_{X} \alpha & =H, \\
i_{X} d \alpha & =-d H+\left(i_{R} d H\right) \alpha,
\end{aligned}
$$

for a choice of $\alpha$ and a function $H \in C^{\infty}(M, \mathbb{R})$. Such a function is called the Hamiltonian associated to the vector field. This correspondance defines a linear isomorphism between the space of vector fields $\Gamma_{\xi}(T M)$ preserving the contact structure $\xi$ and the vector space of smooth functions $C^{\infty}(M, \mathbb{R})$. By definition, a contactomorphism $\phi \in \operatorname{Cont}_{0}(M, \xi)$ admits an expression as $\phi=\phi_{1}$ for a time dependent flow $\left\{\phi_{t}\right\}_{t \in[0,1]}$ generated by a time dependent family $X_{t} \in \Gamma_{\xi}(T M)$. Therefore, its flow $\left\{\phi_{t}\right\}$ can be generated by a time dependent family of smooth functions $\left\{H_{t}\right\}$.

1.2. Contact fibrations. A smooth fibration $\pi: X \longrightarrow B$ is said to be contact for a codimension-1 distribution $\xi \subset T X$ if for any fiber $F_{p}=\pi^{-1}(p) \stackrel{e}{\hookrightarrow} X$, the restriction of the distribution $e^{*} \xi$ is a contact structure on the fibre. We assume that the distribution $\xi$ is cooriented. Any $\alpha \in \Omega^{1}(X)$ such that $\xi=\operatorname{ker} \alpha$ will be referred to as a fibration form.

Let $\pi: X \longrightarrow B$ be a smooth fibration. The vertical subbundle $V \subset T X$ is defined fiberwise by $V_{x}=\operatorname{ker} d \pi(x), \forall x \in X$. An Ehresmann connection is a smooth choice of a fiberwise complementary linear space $H_{x}$ for $V_{x}$ inside $T_{x} X$. Therefore, the map $d \pi_{x}: H_{x} \longrightarrow T B_{\pi(x)}$ is a linear isomorphism and there is a well-defined notion of parallel transport.

There is a canonical connection once a contact fibration $(\pi, \xi=\operatorname{ker} \alpha)$ is fixed. The connection $H$ is defined at a point $x \in X$ to be the annihilator of the vector subspace $V_{x} \cap \xi_{x}$ with respect to the quadratic form $(\xi, d \alpha)$. It is complementary to $V_{x}$ since $V_{x} \cap \xi_{x}$ is a symplectic space for the 2-form $d \alpha$. The connection is independent of the choice of fibration form $\alpha$. See $[\operatorname{Pr}]$ for details on the following facts.

Lemma 4. The parallel transport of the canonical connection associated to a contact fibration is by contactomorphisms.

Lemma 5. Let $\left(F, \operatorname{ker} \alpha_{0}\right)$ be a closed contact manifold. Let $\pi: F \times \mathbb{D}^{2} \longrightarrow \mathbb{D}^{2}$ be a contact fibration with fibration distribution defined by the kernel of $\alpha=\alpha_{0}+H d \theta$, for some function $H: F \times \mathbb{D}^{2} \longrightarrow \mathbb{R}$ satisfying $|H|=O\left(r^{2}\right)$. Fix a loop $\gamma: \mathbb{S}^{1} \longrightarrow \mathbb{D}^{2}$, defined as $\gamma(\theta)=\gamma\left(r_{0}, \theta\right)$ in polar coordinates. Then, the contactomorphism of the fiber $F \times\left(r_{0}, 0\right)$ defined by the parallel transport along $\gamma$ is generated by the family of Hamiltonian functions $\left\{G_{\theta}(p)=-H\left(p, r_{0}, \theta\right)\right\}_{\theta \in[0,2 \pi]}$.

Let us study general contact fibrations over a 2-disk $\mathbb{D}^{2}$. Fix a contact fibration $\pi: X \longrightarrow \mathbb{D}^{2}$ with distribution $\xi=\operatorname{ker} \alpha$. Consider the radial vector field $Y=\partial_{r}$, defined on $\mathbb{D}^{2} \backslash\{0\}$. It can be lifted to $X$ by using the canonical contact connection. This produces a vector field $\tilde{Y}: X \backslash F_{0} \longrightarrow T X$. Once an angle $\theta_{0}$ is fixed it can be uniquely extended to $0 \in \mathbb{D}^{2}$. In such a case, denote by $\phi_{r, \theta_{0}}: F_{0} \longrightarrow F_{\left(r, \theta_{0}\right)}$ the associated flow at time $r$. It identifies via contactomorphisms the fibers over $0 \in \mathbb{D}^{2}$ and over $\left(r, \theta_{0}\right) \in \mathbb{D}^{2}$. Define the diffeomorphism:

$$
\begin{aligned}
\Phi: F_{0} \times D^{2} & \longrightarrow X \\
(p, r, \theta) & \longmapsto \phi_{r, \theta}(p) .
\end{aligned}
$$

Then the definition of the contact connection implies $\Phi^{*} \alpha=e^{g}\left(\alpha_{0}+H d \theta\right)$, where $g: M \times D^{2} \longrightarrow \mathbb{R}$ and $H: M \times D^{2} \longrightarrow \mathbb{R}$ are arbitrary smooth functions. We can choose as fibration form $\alpha^{\prime}=e^{-g} \alpha$ and trivialize the fibration using $\Phi$. Then we obtain the expression

$$
\Phi^{*} \alpha^{\prime}=\left(\alpha_{0}+H d \theta\right) \text {. }
$$

Given a contact fibration over the disk, the trivialization constructed above is called radial. It is convenient to observe that the radial trivialization construction can be made parametric for families of contact fibrations over the disk. 
1.3. Loops at infinity. Fix a contact fibration $\pi: X \longrightarrow \mathbb{S}^{2}$ with distribution $\xi$, fibre $F$ and a point $N \in \mathbb{S}^{2}$. This point will be referred to as North pole or infinity. Define the restriction fibration $\pi_{N}: X \backslash \pi^{-1}(N) \longrightarrow \mathbb{S}^{2} \backslash N \simeq \mathbb{D}^{2}$. Trivialize the contact fibration $\pi_{N}$ radially from $S=\{0\} \in \mathbb{D}^{2}$ to obtain a new contact fibration $\hat{\pi}: F \times \mathbb{D}^{2} \longrightarrow \mathbb{D}^{2}$ with fibration form $\alpha_{0}+H d \theta$. Denoting by $\Phi: F \times \mathbb{D}^{2} \longrightarrow X \backslash \pi^{-1}(N)$ the trivialization map, we obtain $\Phi^{*} \xi=\operatorname{ker}\left\{\alpha_{0}+H d \theta\right\}$. Therefore, the map is connection-preserving. Consider the family of loops

$$
\begin{aligned}
\gamma_{r}: \mathbb{S}^{1} & \longrightarrow D^{2} \\
\theta & \longmapsto(r, \theta) .
\end{aligned}
$$

Composing with the embedding $\mathbb{D}^{2} \hookrightarrow \mathbb{S}^{2}$, for $r \longrightarrow 1$, they are smaller and smaller loops around the North pole $N \in \mathbb{S}^{2}$. By Lemma 5 , the parallel transport associated to the loop $\gamma_{r}$ is generated by a family of Hamiltonians $\left\{G_{\theta}^{r}\right\}_{\theta \in \mathbb{S}^{1}}$, defined by $G_{\theta}^{r}(p)=-H(p, r, \theta)$. The limit function

$$
G_{\theta}=\lim _{r \longrightarrow 1} G_{\theta}^{r}
$$

exists because the connection associated to $\xi$ is a smooth connection over $\mathbb{S}^{2}$. It is clear that $\left\{G_{\theta}\right\}$ defines a loop in $\operatorname{Cont}\left(M, \xi_{0}=\operatorname{ker} \alpha_{0}\right)$. This will be called the loop at infinity associated to $(\pi, \xi)$. Continuous families of contact fibrations with marked fibre produce continuous families of loops at infinity.

Definition 6. A contact sphere is a smooth map e $: \mathbb{S}^{2} \longrightarrow \mathcal{C}(M, \xi)$.

There is a canonical contact fibration over $\mathbb{S}^{2}$ associated to any contact sphere $e$. It is defined as

$$
X=M \times \mathbb{S}^{2} \longrightarrow \mathbb{S}^{2}
$$

with the distribution at $(p, z) \in M \times \mathbb{S}^{2}$ being $\xi^{e}(p, z)=e(z)_{p} \oplus T_{z} \mathbb{S}^{2} \subset T_{p} M \oplus T_{z} \mathbb{S}^{2}$.

Denote by $C^{\infty}\left(\mathbb{S}^{2}, \mathcal{C}(M, \xi)\right)$ the space of smooth maps from $\mathbb{S}^{2}$ to $\mathcal{C}(M, \xi)$. The smooth loop space of $\operatorname{Cont}_{0}(M, \xi)$ is denoted as $\Omega\left(\operatorname{Cont}_{0}(M, \xi), i d\right)$.

Lemma 7. The previous construction induces a continuous map

$$
C^{\infty}\left(\mathbb{S}^{2}, \mathcal{C}(M, \xi)\right) \longrightarrow \Omega\left(\operatorname{Cont}_{0}(M, \xi), i d\right) .
$$

Therefore, it provides a morphism

$$
\pi_{2}(\mathcal{C}(M, \xi)) \longrightarrow \pi_{1}\left(\operatorname{Cont}_{0}(M, \xi)\right) .
$$

1.4. Homotopy sequence. The group $\operatorname{Diff}_{0}(M)$ acts transitively on $\mathcal{C}(M, \xi)$ because of Gray's Stability Theorem. It is a Serre fibration with homotopy fibre $\operatorname{Cont}(M, \xi) \cap \operatorname{Diff}_{0}(M)$. This homotopy fibre might be disconnected. Its identity component is denoted by $\operatorname{Cont}_{0}(M, \xi)$. Hence the fibration induces a long exact sequence

$$
\ldots \longrightarrow \pi_{2}\left(\operatorname{Diff}_{0}(M)\right) \longrightarrow \pi_{2}(\mathcal{C}(M, \xi)) \stackrel{\partial_{2}}{\longrightarrow} \pi_{1}\left(\operatorname{Cont}_{0}(M, \xi)\right) \longrightarrow \pi_{1}\left(\operatorname{Diff}_{0}(M)\right) \longrightarrow \ldots
$$

The map $\partial_{2}$ is the one provided by Lemma 7 . The study of this sequence will provide Corollary 2 .

Note that a geometric lifting map

$$
\pi_{j}(\mathcal{C}(M, \xi)) \stackrel{\partial_{j}}{\longrightarrow} \pi_{j-1}\left(\operatorname{Cont}_{0}(M, \xi)\right)
$$

can be analogously defined. It provides a geometric representative of the connecting morphism. This generalizes the previous constructions. It will be used in Section 4 .

\section{Spheres in $\mathcal{C}(M, \xi)$}

2.1. Almost contact structures. Let $M$ be an oriented $(2 n+1)$-dimensional manifold. Denote by $\operatorname{Dist}(M)$ the space of smooth codimension-1 regular cooriented distributions on $M$. Concerning orientations, an almost complex structure on a cooriented distribution will be positive if the induced orientation coincides with the prescribed one. Define the space of almost contact structures as

$$
\mathcal{A}(M)=\left\{(\xi, \mathfrak{j}): \xi \in \operatorname{Dist}(M), \mathfrak{j} \in \operatorname{End}(\xi), \mathfrak{j}^{2}=-\mathrm{id}, \mathfrak{j} \text { positive }\right\} .
$$


Given a contact structure $\xi=\operatorname{ker} \alpha$, an almost complex structure $\mathbf{j} \in \operatorname{End}(\xi)$ is said to be compatible with $\alpha$ if it is compatible with the symplectic form on the symplectic space $(\xi, d \alpha)$. The space $\mathcal{A}(M)$ has a subset defined by

$$
\mathcal{A C}(M, \xi)=\left\{(\eta, \mathfrak{j}): \eta \in \mathcal{C}(M, \xi), \mathfrak{j} \in \operatorname{End}(\eta), \mathfrak{j}^{2}=-\mathrm{id}, \mathfrak{j} \text { compatible with } \alpha \text { such that } \eta=\text { ker } \alpha\right\} .
$$

The space of almost complex structures compatible with a fixed symplectic form is contractible. Thus, the forgetful map $\mathcal{A C}(M, \xi) \longrightarrow \mathcal{C}(M, \xi)$ has a contractible homotopy fibre. Hence there exists a homotopy inverse $1: \mathcal{C}(M, \xi) \longrightarrow \mathcal{A C}(M, \xi)$ provided by the choice of a compatible almost complex structure on the contact distribution.

Fix a point $p \in M$ and an oriented framing $\tau: T_{p} M \stackrel{\simeq}{\longrightarrow} \mathbb{R}^{2 n+1}$. Define the evaluation map

$$
e_{(p, \tau)}: \mathcal{A}(M) \longrightarrow \mathcal{A}\left(\mathbb{R}^{2 n+1}\right), \quad e_{(p, \tau)}(\xi, \mathfrak{j})=\left(\tau_{*} \xi_{p}, \tau_{*} \mathfrak{j}_{p}\right)
$$

This is a continuous map and thus induces $\widetilde{e}_{(p, \tau)}: \pi_{2}(\mathcal{A}(M)) \longrightarrow \pi_{2}\left(\mathcal{A}\left(\mathbb{R}^{2 n+1}\right)\right)$. Therefore, we obtain

$$
\varepsilon_{(p, \tau)}=\widetilde{e}_{(p, \tau)} \circ 1_{*}: \pi_{2}(\mathcal{C}(M, \xi)) \longrightarrow \pi_{2}\left(\mathcal{A}\left(\mathbb{R}^{2 n+1}\right)\right)
$$

Lemma 8. $\pi_{2}\left(\mathcal{A}\left(\mathbb{R}^{2 n+1}\right)\right) \cong \mathbb{Z}$.

Proof. The space $\mathcal{A}\left(\mathbb{R}^{2 n+1}\right)$ is isomorphic to the homogeneous space $S O(2 n+1) / U(n)$. The standard inclusion $S O(2 n) \longrightarrow S O(2 n+1)$ descends to a map

$$
S O(2 n) / U(n) \longrightarrow S O(2 n+1) / U(n)
$$

with homotopy fibre $\mathbb{S}^{2 n}$. The long exact sequence for a homotopy fibration implies that

$$
\pi_{2}(S O(2 n) / U(n)) \cong \pi_{2}(S O(2 n+1) / U(n)), \quad n \geq 2
$$

It is simple to show that $S O(2 n+1) / U(n)$ is also isomorphic to $S O(2 n+2) / U(n+1)$. Since $S O(4) / U(2)$ is a 2-sphere, the statement follows.

Thus the evaluation map can be seen as an integer-valued map for $\pi_{2}(\mathcal{C}(M, \xi))$.

Lemma 9. The map $\varepsilon_{(p, \tau)}: \pi_{2}(\mathcal{C}(M, \xi)) \longrightarrow \pi_{2}\left(\mathcal{A}\left(\mathbb{R}^{2 n+1}\right)\right)$ is independent of the choice of $p$ and $\tau$.

Proof. Let $p, q \in M$ and $\tau_{p}, \tau_{q}$ be oriented framings of $T_{p} M, T_{q} M$ respectively. Consider a continuous path of pairs $\left\{\left(p_{t}, \tau_{t}\right)\right\}$ connecting $\left(p, \tau_{p}\right)$ and $\left(q, \tau_{q}\right)$. The continuous family of maps

$$
e_{\left(p_{t}, \tau_{t}\right)}: \mathcal{A}(M) \longrightarrow \mathcal{A}\left(\mathbb{R}^{2 n+1}\right), \quad e_{\left(p_{t}, \tau_{t}\right)}(\xi, \mathfrak{j})=\left(\tau_{t_{*}} \xi_{p}, \tau_{t_{*}} \mathfrak{j}_{p}\right)
$$

provides a homotopy between $e_{\left(p, \tau_{p}\right)}$ and $e_{\left(q, \tau_{q}\right)}$.

\subsection{Linear Contact Spheres.}

Definition 10. A linear contact sphere is a contact sphere $\iota: \mathbb{S}^{2} \longrightarrow \mathcal{C}(M, \xi)$ such that there exist three contact forms $\left(\alpha_{0}, \alpha_{1}, \alpha_{2}\right)$ satisfying

$$
\iota(p)=\operatorname{ker}\left(e_{0} \alpha_{0}+e_{1} \alpha_{1}+e_{2} \alpha_{2}\right)
$$

for the standard embedding $\left(e_{0}, e_{1}, e_{2}\right): \mathbb{S}^{2} \longrightarrow \mathbb{R}^{3}$.

Remark 11. Such spheres can only exist in a $(4 n+3)$-dimensional manifold. The fact that $\alpha$ and $-\alpha$ do not induce the same volume form in dimensions congruent to 1 modulo 4 yields an obstruction for their existence.

Note that for a 3-fold the triple $\left(\alpha_{0}, \alpha_{1}, \alpha_{2}\right)$ constitutes a framing of the cotangent bundle.

Lemma 12. Let $M$ be a 3 -fold and $S$ a linear contact sphere. The class $[S] \in \pi_{2}(\mathcal{C}(M, \xi))$ is nontrivial and has infinite order.

Proof. Let $p \in M$ be a point and consider the framing $\tau=\left(\alpha_{0}, \alpha_{1}, \alpha_{2}\right)_{p}$. In the three-dimensional case $\mathcal{A}\left(\mathbb{R}^{3}\right)$ is homotopic to a 2 -sphere. This homotopy can be realized by projection $\pi$ onto the space of cooriented 2-plane distributions. The degree of the evaluation map is computed via

$$
\begin{gathered}
\mathbb{S}^{2} \stackrel{\varepsilon_{(p, \tau)}}{\longrightarrow} \mathcal{A}\left(T_{p} M\right) \stackrel{\pi}{\longrightarrow} \operatorname{Dist}\left(\mathbb{R}^{3}\right) \cong \mathbb{S}^{2} \\
z \longmapsto e_{0}(z) \alpha_{0}(p)+e_{1}(z) \alpha_{1}(p)+e_{2}(z) \alpha_{2}(p) \longmapsto\left(e_{0}(z), e_{1}(z), e_{2}(z)\right) .
\end{gathered}
$$

Being the identity, this map has degree 1. 
2.3. 3-Sasakian manifolds. Let us define a class of contact manifolds with natural linear contact spheres.

Definition 13. Let $\left(M^{4 n+3}, g\right)$ be a Riemannian manifold. It is said to be $3-$ Sasakian if the holonomy group of the metric cone $(C(M), \bar{g})=\left(M \times \mathbb{R}^{+}, r^{2} g+d r \otimes d r\right)$ reduces to $S p(n+1)$.

This implies that $(C(M), \bar{g})$ is a hyperkähler manifold $(C(M), \bar{g}, I, J, K)$. The hyperkähler structure induces a 2 -sphere of complex structures

$$
\mathbb{S}^{2}(\bar{g})=\left\{e_{0} I+e_{1} J+e_{2} K: e_{0}^{2}+e_{1}^{2}+e_{2}^{2}=1\right\} .
$$

Any such complex structure $\mathfrak{j} \in \mathbb{S}^{2}(\bar{g})$ endows $\left(M \times \mathbb{R}^{+}, \bar{g}\right)$ with a Kähler structure, providing $(M, g)$ with a Sasakian structure. The vertical vector field $\partial_{r}$ on $M \times \mathbb{R}^{+}$is orthogonal to $M \times\{1\}$ and the form $\alpha$ defined by $\alpha_{\mathfrak{j}}(v)=g\left(v, \mathfrak{j} \partial_{r}\right)$ is a contact structure. Thus, a 3-Sasakian structure provides a linear contact sphere $\left\{\alpha_{\mathfrak{j}}\right\}_{\mathbf{j} \in \mathbb{S}^{2}(\bar{g})}$ generated by $\alpha_{I}, \alpha_{J}$ and $\alpha_{K}$.

Theorem 14. Let $M^{4 n+3}$ be a 3-Sasakian manifold. The class of the associated linear contact sphere is an element of infinite order in $\pi_{2}\left(\mathcal{C}\left(M, \operatorname{ker}\left(\alpha_{I}\right)\right)\right)$.

Proof. Let $p \in M$ and note that the $4 n$-distribution $\eta=\operatorname{ker} \alpha_{I} \cap \operatorname{ker} \alpha_{J} \cap \alpha_{K}$ is $(I, J, K)$-invariant. Thus, it can be identified with the quaternionic vector space $\mathbb{H}^{n}$ by fixing a quaternionic framing $v=\left\{v_{1}, \ldots, v_{n}\right\}$. This induces a real framing $\tau=\{v, I v, J v, K v\}$ for $\eta$, identifying it with $\mathbb{R}^{4 n}$ endowed with the standard quaternionic structure.

Consider the Reeb vector fields $R_{I}, R_{J}, R_{K}$ associated to $\alpha_{I}, \alpha_{J}$, and $\alpha_{K}$. Extend the framing $\tau$ to $\widetilde{\tau}=\left\{\tau, R_{I}, R_{J}, R_{K}\right\}$. Interpret the space $\mathcal{A}\left(\mathbb{R}^{4 n+3}\right)$ as pairs of $(v, \mathfrak{j})$, where $v \in \mathbb{S}^{4 n+2} \subset \mathbb{R}^{4 n+3}$ is a unit vector and $\mathfrak{j}$ an almost complex structure in its orthogonal space. Define

$$
h: \mathcal{A}\left(\mathbb{R}^{4 n+3}\right) \longrightarrow \mathcal{J}\left(\mathbb{R}^{4 n+3} \oplus \mathbb{R}\right), \quad(v, \mathfrak{j}) \longmapsto\left\{\widetilde{\mathfrak{j}}:\langle v\rangle^{\perp} \oplus\langle v\rangle \oplus\left\langle\partial_{t}\right\rangle \longrightarrow\langle v\rangle^{\perp} \oplus\langle v\rangle \oplus\left\langle\partial_{t}\right\rangle\right\}
$$

where the almost complex structure is $\widetilde{\mathfrak{j}}=\left(\begin{array}{ccc}\mathfrak{j} & 0 & 0 \\ 0 & 0 & -1 \\ 0 & 1 & 0\end{array}\right)$. This induces a morphism of second homotopy groups. Through the above identification the linear contact sphere generated by $\left(\alpha_{I}, \alpha_{J}, \alpha_{K}\right)$ evaluates in a sphere $\left\langle\left(\xi_{I}, I\right),\left(\xi_{J}, J\right),\left(\xi_{K}, K\right)\right\rangle \in \mathcal{A}\left(\mathbb{R}^{4 n+3}\right)$. This sphere maps via (4) to the sphere of complex structures generated by the triple $(I, J, K)$ in $\mathcal{J}\left(\mathbb{R}^{4 n+4}\right)$.

It is left to prove that the class of that sphere is an infinite order element of $\pi_{2}(S O(4 n+4) / U(2 n+2))$. Let us write $m=n+1$ to ease the notation. The homotopy fibration

$$
U(2 m) \longrightarrow S O(4 m) \longrightarrow S O(4 m) / U(2 m)
$$

induces an injection $\pi_{2}(S O(4 m) / U(2 m)) \longrightarrow \pi_{1}(U(2 m)) \cong \mathbb{Z}$.

Let $(\theta, \phi) \in[0,2 \pi] \times[0, \pi]$ be spherical angles. Define

$$
J_{\theta}=\cos \theta J+\sin \theta K, \quad \widetilde{I}=\cos \phi I+\sin \phi J_{\theta}, \quad P_{\theta, \phi}=\cos (\phi / 2) I+\sin (\phi / 2) J_{\theta} .
$$

The sphere is represented by $\widetilde{I}$, we shall compute its image under the boundary morphism. Note that $P_{\theta, \phi} \in S O(4 m)$ and $\widetilde{I}=P_{\theta, \phi}^{t} I P_{\theta, \phi}$. Further $P_{\theta, \pi}=J_{\theta}=(\cos \theta \cdot i d+\sin \theta I) J$, with $\cos \theta \cdot i d+\sin \theta I \in$ $U(2 m)$ and $J \in S O(4 m)$. This decomposition provides a representative in $\pi_{2}(S O(4 m) / U(2 m))$. Thus the loop in $\pi_{1}(U(2 m))$ is provided by $\cos \theta \cdot i d+\sin \theta I$ with $\theta \in[0,2 \pi]$. Since the identification $\pi_{1}(U(2 m)) \cong \mathbb{Z}$ is given by the complex determinant, the degree of the sphere is $2 m$.

The argument above applies to a broader class of manifolds:

Definition 15. A contact manifold $\left(M, \xi_{0}\right)$ is said to possess an almost-quaternionic sphere if it admits a sphere $\mathbb{S}^{2} \stackrel{\xi}{\longrightarrow} \mathcal{C}\left(M, \xi_{0}\right)$ such that:

1) There exists a family $\left\{\mathfrak{j}_{p}\right\}_{p \in \mathbb{S}^{2}}$ compatible with the contact distributions $\xi_{p}=\xi(p)$,

2) There exists a point $q \in M$ and a framing $\tau$ for $T_{q} M$ such that $e_{q, \tau}\left(\xi\left(\mathbb{S}^{2}\right)\right)$ becomes the linear sphere associated to $\left\langle\left(\xi_{I}, I\right),\left(\xi_{J}, J\right),\left(\xi_{K}, K\right)\right\rangle \in \mathcal{A}\left(\mathbb{R}^{4 n+3}\right)$.

Corollary 16. An almost-quaternionic sphere inside a contact manifold $(M, \xi)$ generates a class of infinite order in $\pi_{2}(\mathcal{C}(M, \xi))$. 


\section{Reeb Flow for Spheres}

Let us prove Corollary 2. The standard contact sphere will be denoted $\left(\mathbb{S}^{2 n+1}, \xi\right)$. The relevant case is that of the spheres $\mathbb{S}^{2 k+1}$ with $k$ odd. Indeed, for the spheres $\mathbb{S}^{2 k+1}$ with $k=2 n$ the Reeb flow is non-trivial in $\pi_{1}(S O(4 n+2)) \hookrightarrow \pi_{1}\left(\operatorname{Diff}_{0}\left(\mathbb{S}^{4 n+1}\right)\right)$. Thus it cannot be contractible in $\operatorname{Cont}_{0}(M, \xi) \subset \operatorname{Diff}_{0}\left(\mathbb{S}^{4 n+1}\right)$. In order to conclude the case $\mathbb{S}^{4 n+3}$ we detail the construction in Sections 1 and 2 .

Consider the endomorphisms $I, J, K$ of $\mathbb{R}^{4(n+1)}$ obtained by direct sum of the corresponding endomorphisms $i, j, k$ of $\mathbb{R}^{4}$, satisfying the quaternionic relations

$$
i^{2}=j^{2}=k^{2}=i j k=-1 .
$$

The endomorphisms $I, J, K$ anti-commute and hence any of their linear combinations is a complex structure. Let $e=\left(e_{0}, e_{1}, e_{2}\right): \mathbb{S}^{2} \longrightarrow \mathbb{R}^{3}$ be the standard embedding of the 2-sphere in Euclidean 3 -space with azimuthal angle $\theta$ and polar angle $\phi$ :

$$
e_{0}=\cos \theta \sin \phi, \quad e_{1}=\sin \theta \sin \phi, \quad e_{2}=\cos \phi, \quad(\theta, \phi) \in[0,2 \pi] \times[0, \pi] .
$$

A complex structure $\mathfrak{j} \in \operatorname{End}\left(\mathbb{R}^{4 n+4}\right)$ induces the real $(4 n+2)$-distribution

$$
\xi_{\mathfrak{j}}=T \mathbb{S}^{4 n+3} \cap \mathbf{j} T \mathbb{S}^{4 n+3}
$$

of $\mathfrak{j}$-complex tangencies on the sphere $\mathbb{S}^{4 n+3}$. There exists a unique, up to scaling, $U(\mathfrak{j}, n)$-invariant 1 -form $\alpha_{\mathfrak{j}}$ such that ker $\alpha_{\mathfrak{j}}=\xi_{\mathfrak{j}}$. It is given by $\alpha(z)=z^{t} \mathfrak{j} d z$. We use the following three 1 -forms

$$
\alpha_{0}=\alpha_{I}, \quad \alpha_{1}=\alpha_{J}, \quad \alpha_{2}=\alpha_{K} .
$$

Their respective Reeb vector fields $R_{0}, R_{1}$ and $R_{2}$ are linearly independent and their flows are given by the family of rotations generated by $I, J$ and $K$. Consider the 1 -form $\alpha=e_{0} \alpha_{0}+e_{1} \alpha_{1}+e_{2} \alpha_{2}$. The form $\alpha$ is a contact form on $\mathbb{S}^{4 n+3}$ for each value of $e$. Although not used in the rest of the article, it is simple to prove the following

Lemma 17. $\left(\mathbb{S}^{2} \times \mathbb{S}^{4 n+3}, \operatorname{ker} \alpha\right)$ is a contact manifold.

Let us compute the loop at infinity for the trivial contact fibration

$$
\mathbb{S}^{2} \times \mathbb{S}^{4 n+3} \longrightarrow \mathbb{S}^{2},(e, p) \longmapsto e .
$$

In the spherical coordinates above, we will obtain the loop at infinity corresponding to $\phi=\pi$. The contact connection allows us to lift a vector field $X$ in the base $\mathbb{S}^{2}$. The lift $\tilde{X}$ is the unique vector field on $\mathbb{S}^{2} \times \mathbb{S}^{4 n+3}$ conforming the two conditions

$$
\alpha(\widetilde{X})=0, \quad d \alpha(\tilde{X}, V)=0, \quad \text { with } V \text { an arbitrary vertical vector field. }
$$

Since uniqueness is provided once a solution is found, the following assertion can be readily verified

Lemma 18. The lift of the polar vector field $\partial_{\phi}$ to the contact connection given by $\alpha$ is

$$
\widetilde{X}_{\phi}=\partial_{\phi}+\frac{1}{2}\left(-\sin \theta R_{0}+\cos \theta R_{1}\right) .
$$

The Hamiltonian will appear once we pull-back the contact form $\alpha$ with the $\pi$-time flow of the lift $\widetilde{X}_{\phi}$. Consider the linear endomorphism $F_{\theta}=\frac{1}{2}(-\sin \theta I+\cos \theta J)$. The flow associated to $\widetilde{X}_{\phi}$ induces a diffeomorphism between the central fibre $\{\phi=0\}$ and the fibre at an arbitrary $\phi$. This diffeomorphism can be expressed as

$$
\varphi_{\phi}: \mathbb{S}^{4 n+3} \longrightarrow \mathbb{S}^{4 n+3}, \quad \varphi(p)=e^{F_{\theta} \phi} p .
$$

This is understood as a map in complex space $\mathbb{C}^{2 n+2}$ restricted to the sphere. The theory explained in Section 1, in particular formula (1), implies that the pull-back will be of the form $\alpha_{2}+H(p, \phi) d \theta$. A computation yields

Lemma 19. $\varphi_{\phi}^{*}(\alpha)=\alpha_{2}+\sin ^{2}(\phi / 2) d \theta$

The loops correspond to the flow of the vector field associated to $G=-\sin ^{2}(\phi / 2)$. The loop at infinity has Hamiltonian $\left.G\right|_{\phi=\pi} \equiv-1$. Thus it is the Reeb flow.

We have geometrically realized the boundary map of the long exact homotopy sequence (2). The non-contractibility of the Reeb flow will follow from an understanding of the contact sphere above and the group $\pi_{2}\left(\operatorname{Diff}_{0}\left(\mathbb{S}^{4 n+3}\right)\right)$. Regarding the former we have the following 
Lemma 20. Let $S$ be the sphere of complex structures

$$
S=\left\{e_{0} I+e_{1} J+e_{2} K: e \in \mathbb{S}^{2}\right\} \subset S O(4 n+4) / U(2 n+2) .
$$

1) $S$ represents a non-trivial element of $\pi_{2}(S O(4 n+4) / U(2 n+2)) \cong \mathbb{Z}$.

2) The image of $S$ in $\mathcal{C}\left(\mathbb{S}^{4 n+3}, \xi\right)$ generates an infinite cyclic subgroup in $\pi_{2}\left(\mathcal{C}\left(\mathbb{S}^{4 n+3}, \xi\right)\right)$.

Proof. Both statements follow from the argument provided in the proof of Theorem 14.

Concerning the group Diff $0\left(\mathbb{S}^{4 n+3}\right)$, the following lemma will suffice.

Lemma 21. $\pi_{2}\left(\operatorname{Diff}\left(\mathbb{S}^{4 n+3}\right)\right) \otimes \mathbb{Q}=0$ for $n \geq 2$.

Proof. This is a result in algebraic topology. Let $\operatorname{Diff}_{0}\left(\mathbb{D}^{l}, \partial\right)$ be the group of diffeomorphisms of the $l$-disk restricting to the identity at the boundary. Note the homotopy equivalence

$$
\operatorname{Diff}_{0}\left(\mathbb{S}^{l}\right) \simeq S O(l+1) \times \operatorname{Diff}_{0}\left(\mathbb{D}^{l}, \partial\right)
$$

and that $\pi_{2}(S O(l+1))=0$ since $S O(l+1)$ is a Lie group. Let $\phi(l)=\min \{(l-4) / 3,(l-7) / 2\}$. In the stable concordance range $0 \leq j<\phi(l)$ we have

$$
\pi_{j}\left(\operatorname{Diff}_{0}\left(\mathbb{D}^{l}, \partial\right)\right) \otimes \mathbb{Q}=0, \quad \text { if } l \text { even or } 4 \not j+1 .
$$

See $\left[\right.$ We] for details. In particular $\pi_{2}\left(\operatorname{Diff}_{0}\left(\mathbb{D}^{l}, \partial\right)\right) \otimes \mathbb{Q}=0$ for $l>11$. We are thus able to conclude

$$
\pi_{2}\left(\operatorname{Diff}_{0}\left(\mathbb{S}^{4 n+3}\right)\right) \otimes \mathbb{Q} \cong \pi_{2}\left(\operatorname{Diff}_{0}\left(\mathbb{D}^{4 n+3}, \partial\right)\right) \otimes \mathbb{Q}=0, \quad n>2 .
$$

For the case $n=2$ we provide a more $a d$ hoc argument. Let $C\left(\mathbb{D}^{11}\right)$ be the space of pseudo-isotopies for the disk $\mathbb{D}^{11}$. There exists a homotopy fibration

$$
\operatorname{Diff}_{0}\left(\mathbb{D}^{12}, \partial\right) \longrightarrow C\left(\mathbb{D}^{11}\right) \longrightarrow \operatorname{Diff}_{0}\left(\mathbb{D}^{11}, \partial\right)
$$

Algebraic $K$-theory implies $\pi_{1} C\left(\mathbb{D}^{11}\right) \otimes \mathbb{Q}=\pi_{2} C\left(\mathbb{D}^{11}\right) \otimes \mathbb{Q}=0$. Observe that (5) implies that $\pi_{1}\left(\operatorname{Diff}\left(\mathbb{D}^{12}, \partial\right)\right)$ is a torsion group. The long exact homotopy sequence of the above fibration gives

$$
\ldots \longrightarrow \pi_{2}\left(C\left(\mathbb{D}^{11}\right)\right) \stackrel{\rho_{2}}{\longrightarrow} \pi_{2}\left(\operatorname{Diff}_{0}\left(\mathbb{D}^{11}, \partial\right)\right) \stackrel{\partial}{\longrightarrow} \pi_{1}\left(\operatorname{Diff}_{0}\left(\mathbb{D}^{12}, \partial\right)\right) \stackrel{i_{1}}{\longrightarrow} \pi_{1}\left(C\left(\mathbb{D}^{11}\right)\right) \longrightarrow \ldots
$$

This implies the short exact sequence of Abelian groups

$$
0 \longrightarrow A \longrightarrow \pi_{2}\left(\operatorname{Diff}_{0}\left(\mathbb{D}^{11}, \partial\right)\right) \longrightarrow B \longrightarrow 0,
$$

where $A=\operatorname{ker} \partial=\operatorname{im} \rho_{2}$ and $B=\operatorname{im} i_{1}=\operatorname{coker} \rho_{2}$. Thus $\pi_{2}\left(\operatorname{Diff}_{0}\left(\mathbb{D}^{11}, \partial\right)\right)$ is a torsion group.

Remark 22. The Smale conjeture $\operatorname{Diff}_{0}\left(\mathbb{S}^{3}\right) \simeq S O(4)$ holds for $\mathbb{S}^{3}$, see $[\mathrm{Ha}]$.

In order to conclude Corollary 2 for $\mathbb{S}^{4 n+3}$ consider the class of the Reeb loop in $\pi_{1}\left(\operatorname{Cont}_{0}\left(\mathbb{S}^{4 n+3}, \xi\right)\right)$. The construction explained above shows that it lies in the image of the boundary morphism

$$
\partial_{2}: \pi_{2}\left(\mathcal{C}\left(\mathbb{S}^{4 n+3}, \xi\right)\right) \longrightarrow \pi_{1}\left(\operatorname{Cont}_{0}\left(\mathbb{S}^{4 n+3}, \xi\right)\right) .
$$

If the Reeb class were to be zero the sphere $S$ would lie in the image of $\pi_{2}\left(\operatorname{Diff}_{0}\left(\mathbb{S}^{4 n+3}\right)\right)$ in $(2)$. Lemma 21 implies that such a sphere needs to be a torsion class if $n \geq 2$. Lemma 20 contradicts this statement. Thus proving Corollary 2.

\section{HigheR HOMOTOPY GROUPS}

The previous arguments can be modified for $n$-dimensional homotopy spheres. This allows us to conclude properties of the higher homotopy type of the contactomorphism group. Consider the evalution map

$$
e_{p, \tau}: \mathcal{A}(M) \longrightarrow \mathcal{A}\left(\mathbb{R}^{2 n+1}\right) .
$$

Composition with the homotopy inverse $1: \mathcal{C}(M) \rightarrow \mathcal{A C}(M)$ defines higher homotopy maps

$$
\pi_{k}\left(e_{p, \tau} \circ 1\right): \pi_{k}(\mathcal{C}(M)) \longrightarrow \pi_{k}\left(\mathcal{A}\left(\mathbb{R}^{2 n+1}\right)\right), \quad k \geq 1 .
$$

Let us provide a simple application. Define the natural inclusion

$$
i_{\mathcal{J}}: \mathcal{J}\left(\mathbb{R}^{2 n+2}\right) \longrightarrow \mathcal{C}\left(\mathbb{S}^{2 n+1}, \xi\right), \quad i_{\mathcal{J}}(\mathfrak{j})=T \mathbb{S}^{2 n+1} \cap \mathfrak{j} T \mathbb{S}^{2 n+1} .
$$

Lemma 23. The map $i_{\mathcal{J}}$ is a homotopy inclusion. 
Proof. Consider the following chain of maps

$$
c: \mathcal{J}\left(\mathbb{R}^{2 n+2}\right) \stackrel{i_{\mathcal{J}}}{\longrightarrow} \mathcal{C}\left(\mathbb{S}^{2 n+1}, \xi\right) \stackrel{e_{p, \tau} \text { o1 }}{\longrightarrow} \mathcal{A}\left(\mathbb{R}^{2 n+1}\right) \stackrel{h}{\longrightarrow} \mathcal{J}\left(\mathbb{R}^{2 n+2}\right) .
$$

The definition of each map implies $c=i d$. Therefore, it induces the identity in homotopy:

$$
\pi_{k}(c)=i d: \pi_{k}\left(\mathcal{J}\left(\mathbb{R}^{2 n+2}\right)\right) \stackrel{\pi_{k}\left(i_{\mathcal{J}}\right)}{\longrightarrow} \pi_{k}\left(\mathcal{C}\left(\mathbb{S}^{2 n+1}\right), \xi\right) \stackrel{\pi_{k}\left(e_{p, \tau} \circ 1\right)}{\longrightarrow} \pi_{k}\left(\mathcal{A}\left(\mathbb{R}^{2 n+1}\right)\right) \stackrel{\pi_{k}(h)}{\longrightarrow} \pi_{k}\left(\mathcal{J}\left(\mathbb{R}^{2 n+2}\right)\right) .
$$

Thus the map $i_{\mathcal{J}}$ induces an injection $\pi_{k}\left(i_{\mathcal{J}}\right), \forall k \geq 0$.

This lemma can be combined with results on the homotopy type of the group Diff $\left(\mathbb{S}^{2 n+1}\right)$. We can then conclude the existence of infinite order elements in certain homotopy groups of $\operatorname{Cont}\left(\mathbb{S}^{2 n+1}, \xi\right)$. Among many others, a simple instance is the following

Lemma 24. The group $\pi_{5}\left(\operatorname{Cont}\left(\mathbb{S}^{2 n-1}, \xi\right)\right)$ has an element of infinite order, for $n \geq 12$.

Proof. Using the connecting map $\partial_{6}$, as described in equation (3), the statement is reduced to the following two assertions:

- $\pi_{6}\left(\mathcal{J}\left(\mathbb{R}^{2 n}\right)\right)=\pi_{6}(S O(2 n) / U(n))=\mathbb{Z}$ and therefore, by Lemma $23, \operatorname{rk}\left(\pi_{6}\left(\mathcal{C}\left(\mathbb{S}^{2 n-1}, \xi\right)\right)\right) \geq 1$.

- $\pi_{6}\left(\operatorname{Diff}\left(\mathbb{S}^{2 n-1}\right)\right) \otimes \mathbb{Q}=0$, for $n \geq 12$. This is again a consequence of the results in [We].

Bott Periodicity Theorem allows us to apply the same argument to infinitely many other homotopy groups of Cont $\left(\mathbb{S}^{2 n-1}, \xi\right)$. These techniques can be adapted for general contact manifolds as long as there is a partial understanding of the homotopy type of their group of diffeomorphisms.

\section{REFERENCES}

[BG] C.P. Boyer, K. Galicki, 3-Sasakian manifolds, Surveys in Differential Geometry: Essays on Einstein Manifolds, Surv. Differ. Geom. VI. Boston Int. Press (1999), 123-184.

[Bo] F. Bourgeois, Contact homology and homotopy groups of the space of contact structures, Math. Res. Lett. 13 (2006), 71-85.

[El] Y. Eliashberg, Contact 3-manifolds twenty years since J. Martinet's work, Ann. Inst. Fourier 46 (1992), $165-192$.

[Ge] H. Geiges, J. Gonzalo, On the topology of the space of contact structures on torus bundles, Bull. London Math. Soc. 36 (2004), 640-646.

[Gi] E. Giroux, Sur la géométrie et la dynamique des transformations de contact, Séminaire Bourbaki (2009), n.1004.

[Ha] A. E. Hatcher, A proof of the Smale conjecture, Ann. of Math. 117 (1983), 553-607.

[Pr] F. Presas, A class of non-fillable contact structures, Geometry\&Topology 11 (2007), 2203-2225.

[We] M. Weiss, B. Williams, Automorphisms of manifolds, Surveys on Surgery Theory. Vol. II Papers Dedicated to C.T.C Wall, Annals of Mathematics Studies 149 (2001).

Instituto de Ciencias Matemáticas - CSiC. C. Nicolás Cabrera, 13-15, 28049, Madrid, Spain.

E-mail address: casals.roger@icmat.es

Instituto de Ciencias Matemáticas - CSiC. C. Nicolás Cabrera, 13-15, 28049, Madrid, Spain.

E-mail address: fpresas@icmat.es 